\title{
Modulus of orbital analytic classification for a family unfolding a saddle-node
}

\author{
Christiane Rousseau*
}

CRM-2915

March 2003

\footnotetext{
* Département de mathématiques et de statistique and CRM, Université de Montréal, C.P. 6128, Succursale Centre-ville, Montréal (Qué.), H3C 3J7, Canada
} 



\begin{abstract}
In this paper we consider generic families of 2-dimensional analytic vector fields unfolding a generic (codimension 1) saddle-node at the origin. We show that a complete modulus of orbital analytic classification for the family is given by an unfolding of the Martinet-Ramis modulus of the saddle-node. The Martinet-Ramis modulus is given by a pair of germs of diffeomorphisms, one of which is an affine map. We show that the unfolding of this diffeomorphism in the modulus of the family is again an affine map. The point of view taken is to compare the family with the "model family" $\left(x^{2}-\epsilon\right) \frac{\partial}{\partial x}+y(1+a(\epsilon) x) \frac{\partial}{\partial y}$. The nontriviality of the Martinet-Ramis modulus implies geometric "pathologies" for the perturbed vector fields, in the sense that the deformed family does not behave as the standard family.
\end{abstract}

\title{
Résumé
}

Dans cet article nous considérons des familles génériques de champs de vecteurs bi-dimensionnels déployant un col-nœud générique (de codimension 1) à l'origine. On montre qu'un module complet de classification analytique orbitale pour la famille est donné par un déploiement du module de Martinet-Ramis pour le col-nœud. Le module de Martinet-Ramis est donné par une paire de germes de difféomorphismes, dont l'un est une transformation affine. Nous montrons que son déploiement dans le module de la famille reste une trasformation affine. Le point de vue utilisé est de comparer la famille avec la "famille modèle" $\left(x^{2}-\epsilon\right) \frac{\partial}{\partial x}+y(1+a(\epsilon) x) \frac{\partial}{\partial y}$. La non trivialité du module de Martinet-Ramis inplique des "pathologies" géométriques pour les champs de vecteurs perturbés, dans le sens que la famille considérée ne se comporte pas comme la famille standard. 



\section{Introduction}

A vector field with a codimension 1 saddle-node at the origin is formally orbitally equivalent to the vector field

$$
x^{2} \frac{\partial}{\partial x}+y(1+a x) \frac{\partial}{\partial y}
$$

which we call the "model", but generically the change of coordinates and scaling of time to the form (1.1) diverges. Why? Because the geometry of the model is simpler than the geometry of the original vector field. The Martinet-Ramis modulus allows to describe the geometry of the foliation of the original vector field. Indeed the space of leafs is described on two open sectors whose union covers a neighborhood of the origin. In the model we have a trivial glueing, while the glueing is non trivial in generic saddle-nodes. The equivalence class of a saddle-node (under orbital equivalence) is completely characterized by the conjugacy class of the holonomy map of its strong separatrix, the later being given by the Ecalle-Voronin modulus of the holonomy map. In general the Ecalle-Voronin of a diffeomorphism having a generic parabolic fixed point at the origin is given by a pair of germs of diffeomorphisms $\left(\psi^{0}, \psi^{\infty}\right)$ defined respectively at the origin and at infinity. In the particular case of the holonomy map of a saddle-node the map $\psi^{\infty}$ is an affine map.

The following remark appears in [6]:

Un phénomène qui reste un peu surprenant à nos yeux est que les holonomies produites par les équations (2) ne sont pas arbitraires: on obtient seulement une "petite partie du module d'Ecalle". (Nous nous proposons de montrer dans un article ultérieur qu'il n'en est plus de même dans le cas des équations résonantes "non dégénérées": le module des classes d'équivalence analytiques d'équations différentielles s'identifie complètement au "module d'Ecalle").

Our study below will in particular provide a full explanation of this surprising phenonmenon. Indeed the best way to understand the geometric meaning of these non trivial glueings is by unfolding the saddlenode. This point of view has already been studied by Glutsyuk in [2] where he studies the unfolding in the Poincaré domain and shows that the Martinet-Ramis modulus is the limit of the transition maps between the linearizing changes of coordinates in the neighborhood of the two singular points. But this does not explain the particular form of one of the diffeomorphisms in the Martinet-Ramis invariant. We push his study further by considering also the unfolding in the Siegel direction when the point is deformed into a saddle and a node.

In this paper we treat the following questions:

(1) We show that a generic family of 2-dimensional vector fields unfolding a saddle-node can be brought by an analytic change of coordinate depending analytically of the parameter $\hat{\epsilon} \neq 0$ on a neighborhood of the origin in the Riemann surface of $\sqrt{\epsilon}$ and continuously on $\hat{\epsilon}$ near $\hat{\epsilon}=0$ to the prenormal form:

$$
\begin{aligned}
& \dot{x}=x^{2}-\epsilon \\
& \dot{y}=\left(x^{2}-\epsilon\right) f_{0}(x)+y(1+a(\epsilon) x)+o(y) .
\end{aligned}
$$

(2) We prove an analytic center manifold theorem for an analytic family (1.2) unfolding a saddle-node: we get a family of invariant manifolds which are analytic at the singular point $(-\sqrt{\hat{\epsilon}}, 0)$ and ramified at $(\sqrt{\hat{\epsilon}}, 0)$, for $\hat{\epsilon}$ is in an open sector of the universal covering of $\epsilon$-space of opening greater than $2 \pi$.

(3) We give a geometric proof of the fact that the map $\psi^{\infty}$ of the Martinet-Ramis modulus is an affine map. Indeed by looking at the geometry of the leaves we show that this map is necessarily a global diffeomorphism of $C P^{1}$ fixing $\infty$. Hence it is an affine map as the affine maps are the only global diffeomorphims of $C P^{1}$ fixing $\infty$.

(4) We show that the unfolded diffeomorphism $\psi_{\epsilon}^{\infty}$ remains global for the unfolding of the saddle-node, yielding that it remains an affine map. 
(5) We exploit this to construct changes of coordinates (ramified at $\pm \sqrt{\epsilon}$ ) transforming the family (1.2) to the model family

$$
\begin{aligned}
\dot{x} & =x^{2}-\epsilon \\
\dot{y} & =y(1+a(\epsilon) x) .
\end{aligned}
$$

This allows to show that the complete modulus of analytic classification for the holonomy map of the family (1.2) (see [8]), given by the family $\left(\psi_{\hat{\epsilon}}^{0}, \psi_{\hat{\epsilon}}^{\infty}\right)_{\hat{\epsilon} \in V}$ where $V$ an open sector of the universal covering of $\epsilon$-space of opening greater than $2 \pi$, is a complete modulus of analytic classification for the family (1.2) under weak orbital equivalence (to be defined below).

(6) The non triviality (i.e. nonlinearity) of $\psi^{\infty}$ implies that in the unfolding the analytic weak separatrix of the saddle is always ramified at the node. The latter implies that the node is non linearizable as soon as resonant. Similarly the non triviality (i.e. nonlinearity) of $\psi^{0}$ implies that the saddle is non integrable for some sequences of parameter values depending on the nonlinearities of $\psi^{0}$ (see also [8]). We call this phenomenon parametric resurgence as the pathologies of the system may only be seen on the types of the singular points at discrete values of the parameters.

(7) We finally treat an example: the Ricatti equation and its subcase: the linear equation. In this particular case we completely calculate $\psi_{\epsilon}^{\infty}$ and show that its coefficient is holomorphic if $a(\epsilon) \equiv n$, $n \in Z$ and holomorphic except at $\epsilon$ where it has an essential singularity otherwise.

This paper is dedicated to Yulij Ilyashenko who first introduced the author to the Martinet-Ramis modulus.

\section{Preparation of the family}

We start with a system which has a generic saddle-node at the origin. Under an analytic change of coordinate we can bring the system to the prenormal form

$$
\begin{aligned}
& \dot{x}=x^{2} \\
& \dot{y}=y(1+a x)+x^{2} f_{0}(x)+x^{2} y^{2} R(x, y) .
\end{aligned}
$$

We consider a generic family unfolding a saddle-node. If the family depends continuously on a parameter $\epsilon$ the following theorem was proved by Glutsyuk.

2.1 Theorem [2] There exists an analytic change of coordinates depending continuously on the parameter $\epsilon$ bringing a generic family unfolding a saddle-node to the prenormal form

$$
\begin{aligned}
& \dot{x}=x^{2}-\epsilon \\
& \dot{y}=\left(x^{2}-\epsilon\right) g_{0}(x)+y(1+g(x, y, \epsilon))
\end{aligned}
$$

where $g(x, y, \epsilon)=O(x)+O(y)$.

We improve his result. We start with a generic family unfolding a saddle-node. This is done in two folds. First we remark that, if we apply the Glutsyuk argument to a family depending analytically on $\epsilon$, the change of coordinate to the form (2.2) is analytic in $\sqrt{\hat{\epsilon}} \neq 0$ and continuous in $\sqrt{\hat{\epsilon}}$ at $\epsilon=0$. We then "prepare" the family so that the parameter becomes an analytic invariant.

2.2 Theorem We consider a generic 1-parameter family unfolding a saddle-node. There exists an analytic change of coordinates depending analytically on $\sqrt{\epsilon} \neq 0$ and continuously on $\sqrt{\epsilon}$ near $\epsilon=0$ bringing the family to the prepared normal form

$$
\begin{aligned}
& \dot{x}=x^{2}-\epsilon \\
& \dot{y}=\left(x^{2}-\epsilon\right) g_{0}(x)+y(1+a(\epsilon) x)+o(y) .
\end{aligned}
$$

In this form the parameter $\epsilon$ and $a(\epsilon)$ are analytic invariants. 
Proof. Glutsyuk first shows that the family can be brought to the form

$$
\begin{aligned}
& \dot{x}=x^{2}-\epsilon+y R(x, y, \epsilon) \\
& \dot{y}=\left(x^{2}-\epsilon\right) g_{0}(x)+y(1+q(x, y, \epsilon)) .
\end{aligned}
$$

For $\epsilon \neq 0$ the strong separatrices of the singular points $( \pm \sqrt{\epsilon}, 0)$ are analytic curves $x=F_{\epsilon}^{ \pm}(y)$ depending analytically on $\sqrt{\epsilon}$. Glutsyuk shows that the graphs of $F_{\epsilon}^{ \pm}$are defined on neighborhoods of zero whose size is independent of $\epsilon$. Then one first performs the change $x_{1}=x-F_{\epsilon}^{+}(y)$ which straightens the separatrix of $(\sqrt{\epsilon}, 0)$. Let $x_{1}=\bar{F}_{\epsilon}^{-}(y)$ be the equation of the separatrix of $(-\sqrt{\epsilon}, 0)$. The next transformation is $x_{2}=-2 x_{1} \frac{\sqrt{\epsilon}}{\bar{F}_{\epsilon}^{-}(y)}$ which preserves the first separatrix and straightens the second one. Making a translation in $x_{2}$ and scaling in $x_{2}$ yields the form (2.4) with the required dependence on the parameter. For the next step we now change $x_{2}=x$.

We now let $q(x, y, \epsilon)=q_{1}(x, \epsilon)+O(y)$. By Kostov's theorem a change of coordinates in $(x, \epsilon) \mapsto(\bar{x}, \bar{\epsilon})$ allows to bring $\frac{x^{2}-\epsilon}{1+q_{1}(x, \epsilon)} \frac{\partial}{\partial x}$ to the form $\frac{\bar{x}^{2}-\bar{\epsilon}}{1+a(\bar{\epsilon})} \frac{\partial}{\partial \bar{x}}$. Applying this to the system $(2.4)$ divided by $1+q(x, \epsilon)$ and then multiplying the obtained system by $1+a(\bar{\epsilon}) \bar{x}$ yields the result, by remarking that in Kostov's proof $\left(\bar{x}^{2}-\bar{\epsilon}\right)$ is a nonzero multiple of $\left(x^{2}-\epsilon\right)$. Let $\mu_{ \pm}= \pm \frac{2 \sqrt{\epsilon}}{1 \pm a(\epsilon) \sqrt{\epsilon}}$ be the quotient of the eigenvalues of $\pm \sqrt{\epsilon}$. Then $\mu_{ \pm}$are analytic invariants of the system. The parameter $\epsilon$ and also $a(\epsilon)$ are analytic invariants since

$$
\begin{aligned}
& \frac{1}{\sqrt{\epsilon}}=\frac{1}{\mu_{+}}-\frac{1}{\mu_{-}} \\
& a(\epsilon)=\frac{1}{\mu_{-}}+\frac{1}{\mu_{+}} .
\end{aligned}
$$

\section{A center manifold theorem for analytic families of vector fields}

3.1 Theorem We consider a germ of family of analytic vector fields of the form

$$
v_{\epsilon}=\left(x^{2}-\epsilon\right) \frac{\partial}{\partial x}+\left[\left(x^{2}-\epsilon\right) f_{0}(x, \epsilon)+y(1+a(\epsilon) x)+\sum_{i \geq 2} f_{i}(x, \epsilon) y^{i}\right] \frac{\partial}{\partial y},
$$

depending analytically on $\sqrt{\epsilon}$ for $\epsilon \neq 0$ and continuously on $\sqrt{\epsilon}$ near $\epsilon=0$. Let $\delta>0$ small. Then there exists a sector $V$ of the universal covering of $C$ :

$$
V=\{\hat{\epsilon} ;|\hat{\epsilon}|<\rho, \arg \hat{\epsilon} \in(-2 \pi+\delta, 2 \pi-\delta)\},
$$

a neighborhood $U$ of the origin in $x$-space and an invariant manifold defined as the graph of a function $y=g_{\hat{\epsilon}}(x)$ defined on a neighborhood $U$ of the origin in $x$-space such that

i) For $\epsilon=0$ the function $g_{0}(x)$ is 1-summable in all directions except in the direction $R^{+}$and its sum is a ramified function over $U$ (Figure 1a).

ii) The function $g_{\hat{\epsilon}}(x)$ depends analytically on $\hat{\epsilon} \neq 0$ and continuously on $\hat{\epsilon}$ near $\epsilon=0$.

iii) The function $g_{\hat{\epsilon}}(x)$ is analytic on $U$ minus a cut from $\sqrt{\epsilon}$.

iv) As a ramified function the function $g_{\hat{\epsilon}}$ is defined over a domain of the form $U_{\hat{\epsilon}}$ projecting on $U$ as in Figure 1b).

Proof. We can scale $y$ and suppose that the system (3.1) is defined over $|y|<2$.

The function $g_{\epsilon}(x)$ of the theorem must be a solution of the nonlinear differential equation:

$$
\left(x^{2}-\epsilon\right) g_{\epsilon}^{\prime}(x)=g_{\epsilon}(x)(1+a x)+\sum_{i \geq 2} f_{i}(x, \epsilon) g_{\epsilon}^{i}(x)+\left(x^{2}-\epsilon\right) f_{0}(x, \epsilon) .
$$




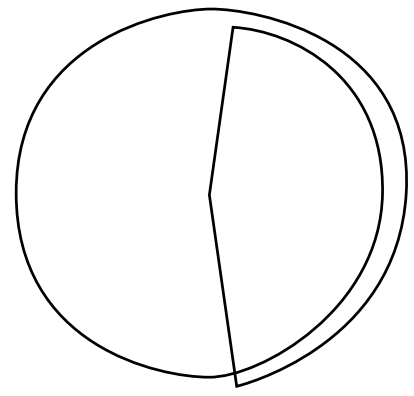

(a) $\epsilon=0$

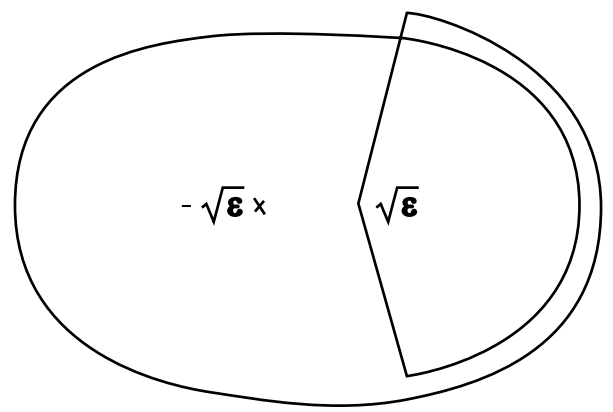

(b) $\hat{\epsilon} \neq 0$

Figure 1: The domain of $g_{\hat{\epsilon}}$

For $\epsilon=0$ the solution of (3.3) is 1-summable in all directions except in the direction $R^{+}$[6]. This yields to the existence of a ramified solution over a domain $U_{0}$ in the universal covering of $C \backslash\{0\}$ as in Figure 1a), the opening of the sector being $(-\pi / 2+\delta, 5 \pi / 2-\delta)$. We take $U_{1}$ to be the projection of $U_{0}$. We suppose that $U_{1}$ contains a disk $\bar{U}=\{|x| \leq r\}$ with interior $U$. Let $0<R<1$. We can always suppose that $r$ is sufficiently small so that $\left|g_{0}(x)\right|<|x|<R$ for $|x|=r$ (this comes from the fact that $g_{0}(x)$ has an asymptotic expansion of the form $O\left(x^{2}\right)$ near $x=0$.

For $\epsilon \in V$ the equation (3.3) has an analytic solution defined in the neighborhood of $-\sqrt{\epsilon}$ and vanishing at $x=-\sqrt{\epsilon}$ (because the quotient of eigenvalues is not a positive real number). For $|\epsilon|$ sufficiently small we now need to extend this solution to the fixed neighborhood $|x| \leq r$, independently of $\epsilon$, with a cut from $x=\sqrt{\epsilon}$. The ideas used here are borrowed from Glutsyuk [2]. Indeed for $(x, \epsilon)$ sufficiently small the inequality $|\dot{y}|>|\dot{x}|$ is satisfied for $(x, y)$ in the cones: $K_{1}(\epsilon)=\{(x, y) ;|y|>|x-\sqrt{\epsilon}|\}$ and $K_{2}(\epsilon)=\{(x, y) ;|y|>|x+\sqrt{\epsilon}|\}$. Also leaves of the foliation of (3.1) contain trajectories with real time of all systems of the form

$$
v_{\epsilon}(\theta)=e^{i \theta} \times v_{\epsilon} .
$$

We need to find points $\left(x_{1}, \bar{g}_{\epsilon}\left(x_{1}\right)\right)$, with $\left|x_{1}\right|=r$, which "should" belong to the center manifold and are located under the cones $K_{1}$ and $K_{2}$. The extension of their trajectories under the different $v_{\epsilon}(\theta)$ will yield the full center manifold. The details are as follows.

We let $x_{0}=-r$. Let $\phi_{0}^{t}$ be the flow of $v_{0}$ (we allow complex time). Then for all $\left(x_{1}, g_{0}\left(x_{1}\right)\right)$ with $\left|x_{1}\right|=r$ and $\arg \left(x_{1}\right) \in(-\pi / 2+\delta, 5 \pi / 2-\delta)$ there exists $t\left(x_{1}\right) \in C$ such that $\left(x_{1}, g_{0}\left(x_{1}\right)\right)=\phi_{0}^{t\left(x_{1}\right)}\left(x_{0}, g_{0}\left(x_{0}\right)\right)\left(t\left(x_{1}\right)\right.$ is multivalued on $\Re x_{1}>0$.)

Let $\eta>0$ small. The trajectories with real time starting at $\left(x_{0}, y\right)$ with $\left|y-g_{0}\left(x_{0}\right)\right|=\eta$, i.e on a circle $\Gamma$, cross the cylinder $C$ given by $|y|=1$ along a non-contractible loop $\gamma$ : this yields a continuous map $P_{0}$ from the circle $\Gamma$ to the cylinder $C$.

We will limit ourselves to values of $|\epsilon|<\rho_{1}$ with $\rho_{1}$ sufficiently small so that $\pm \sqrt{\epsilon}$ remain inside $|x|<r$. For small $\epsilon$ the map $P_{0}$ is deformed to a continuous map $P_{\epsilon}$ from the circle $\Gamma$ to the cylinder $C$. Hence there is a topological obstruction to the continuous extension of $P_{\epsilon}$ to the disk $D=\left\{x=x_{0},\left|y-g_{0}(x)\right| \leq \eta\right\}$ given by the interior of $\Gamma$, yielding that the orbit of at least one point $\left(x_{0}, y_{\epsilon}\right)$ of $D$ does not meet the cylinder.

Then the forward trajectory of $\left(x_{0}, y_{\epsilon}\right)$ "remains under" the cones $K_{1}$ and $K_{2}$, i.e. lies in the region $|y|<\min (|x-\sqrt{\epsilon}|,|x+\sqrt{\epsilon}|)$. We let $\bar{g}_{\epsilon}\left(x_{0}\right)=y_{\epsilon}$.

For all $x_{1}$ with $\left|x_{1}\right|=r$ there exists $t_{\epsilon}\left(x_{1}\right)\left(t\left(x_{1}\right)\right.$ is multivalued on $\left.\Re x_{1}>0\right)$ such that $\phi_{\epsilon}^{t_{\epsilon}\left(x_{1}\right)}\left(x_{0}, y_{\epsilon}\right)=$ $\left(x_{1}, y_{1, \epsilon}\right)$. We let $\bar{g}_{\epsilon}\left(x_{1}\right)=y_{1, \epsilon}$. When $\epsilon$ is small the map $\bar{g}_{\epsilon}$ is close to $g_{0}$ on $|x|=r$.

For all $\hat{\epsilon} \in V$ such that the $x$-eigenvalue of $v_{\hat{\epsilon}}$ at $(-\sqrt{\hat{\epsilon}}, 0)$ has a negative real part (for instance $\arg (\hat{\epsilon}) \in(-\pi+\delta, \pi-\delta))$ at least one of the trajectories of a $v_{\epsilon}(\theta)$ starting at one point $\left(x_{1}, y_{1, \epsilon}\right)$ tends to 
$(-\sqrt{\hat{\epsilon}}, 0)$, yielding that it is part of the invariant manifold of that point. Hence all trajectories starting at points $\left(x_{1}, y_{1, \epsilon}\right)$ belong to the invariant manifold of $(-\sqrt{\hat{\epsilon}}, 0)$, i.e. give an extension of $g_{0}(x)$.

The property follows for the other values of $\hat{\epsilon}$ by analytic extension.

3.2 Theorem We consider a family (3.1) as in Theorem 3.1 which has no analytic center manifold for $\epsilon=0$. Then the function $g_{0}(x)$ is ramified in a nontrivial way at $\sqrt{\epsilon}$ for all $\hat{\epsilon} \in V$. In particular the node of (3.1) located at $(\sqrt{\epsilon}, 0)$ is non linearizable (i.e. has a nonzero resonant monomial) as soon as it is resonant.

Proof. From our proof it follows that if the map $g_{0}$ of Theorem 3.1 is ramified over $|x| \leq r$, then so is the map $g_{\epsilon}$ for small $\epsilon$. The only point where the ramification can occur is $\sqrt{\epsilon}$ as the map is analytic in $x$ elsewhere. The second part follows by noting that all trajectories through a linearizable resonant node are analytic and therefore cannot be ramified. Hence the only possible way to have a non analytic trajectory passing through a resonant node is to have a nonlinearizable node (see also [8]).

\section{The unfolding of the Martinet-Ramis modulus}

\subsection{The Martinet-Ramis modulus of orbital analytic classification for a saddle-node}

The description of the modulus of orbital analytic classification of a saddle-node, called the MartinetRamis modulus makes use of first integrals defined in sectorial neighborhoods of the saddle-node ([6] and [3]). Moreover it is shown in [6] that the orbital analytic class of a saddle-node is characterized by the analytic class of the holonomy of its strong separatrix (see also [3]). Indeed the first integral is a tool to describe the space of orbits of the vector field (leaves of the foliation), the later coinciding (up to isolated elements) with the space of orbits of the holonomy map.

The holonomy map is formally equivalent to the $2 \pi i$-time map of the vector field $\frac{x^{2}}{1+a x} \frac{\partial}{\partial x}$ i.e. the time 1-map of

$$
2 \pi i \frac{x^{2}}{1+a x} \frac{\partial}{\partial x}
$$

(see Figure 2). Taking $\bar{x}=2 \pi i x$ we can also see it as the time one map of

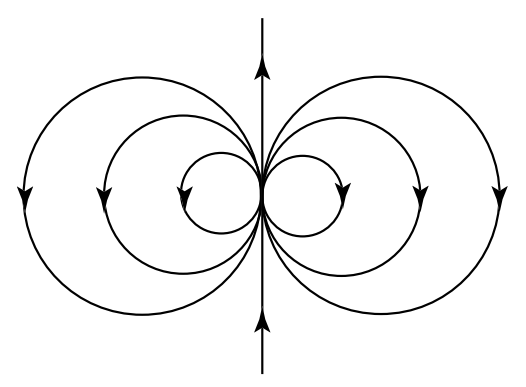

Figure 2: The vector field (4.1)

$$
\frac{\bar{x}^{2}}{1+\overline{a x}} \frac{\partial}{\partial \bar{x}}
$$

with $\bar{a}=\frac{a}{2 \pi i}$.

For the holonomy map the space of orbits is described by two fundamental domains for sectorial neighborhoods $U^{ \pm}$of the origin where $U^{+}\left(\operatorname{resp} U^{-}\right)$is a sector defined by $\arg x \in(-3 \pi / 2+\eta, \pi / 2-\eta)$ (resp. $\arg x \in(-\pi / 2+\eta, 3 \pi / 2-\eta))$ with $\eta \in(0, \pi / 2)$. Once quotiented by the holonomy map, these fundamental domains have the conformal structure of punctured spheres. As orbits may have representatives in the two fundamental domains the spheres are glued in the neighborhoods of zero and infinity by the Ecalle-Voronin 
modulus $\left(\psi^{0}, \psi^{\infty}\right)$, where $\psi^{0}$ and $\psi^{\infty}$ are germs of analytic diffeomorphisms in the neighborhood of 0 and $\infty$ (Figure 3). When unfolding a saddle-node in the Siegel direction we will get essentially a saddle and a

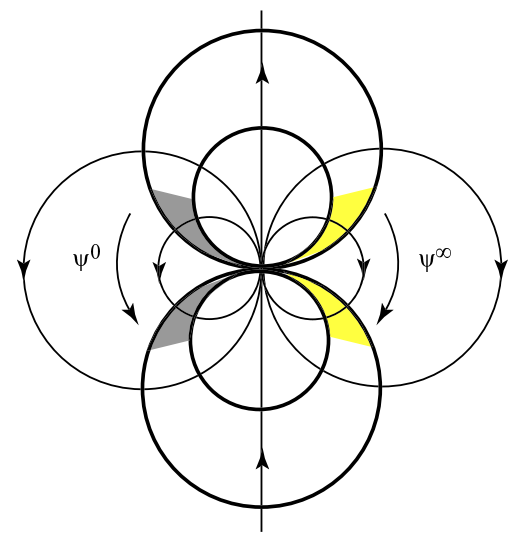

Figure 3: The fundamental domains and transition maps

node ("essentially" because if $a \notin Z$ there may be a small shift between the values of $\epsilon$ for which one point is a saddle and those for which the other singular point is a node). We choose to call $\infty$ (resp. 0) the point of the sphere which will be attached to the node (resp. saddle). The coordinates on the spheres are uniquely determined up to linear transformations on each sphere. Under rescaling of $y$ we can suppose that the holonomy map is defined for a section $\{y=1\}$ : we call it $f_{0}$. All leaves of the foliation intersect this section except possibly one (the center manifold). Each leaf intersects at least one fundamental domain. Hence, it is natural to take the spherical coordinates as first integrals. Then the Ecalle-Voronin modulus represents exactly the transitions between the two first integrals $H_{0}^{ \pm}$defined on $U^{ \pm} \times W$ where $W$ is a neighborhood of the origin in $y$-space. These two first integrals are the "canonical" first integrals

$$
H_{0}^{ \pm}(x, Y)=Y_{ \pm} x^{-a} e^{\frac{1}{x}}
$$

for the model

$$
\begin{aligned}
\dot{x} & =x^{2} \\
\dot{Y} & =Y(1+a x),
\end{aligned}
$$

where $Y_{ \pm}=Y_{ \pm}(x, y)$ are the normalizing coordinates on the two domains $U^{ \pm} \times W$. (See for instance [3]).

In general all pairs of germs of analytic diffeomorphisms $\left(\psi^{0}, \psi^{\infty}\right)$ at the origin and at $\infty$ can be realized as the Ecalle-Voronin modulus of a germ of diffeomorphism with a generic parabolic fixed point or as the Martinet-Ramis modulus of a saddle with eigenvalues 1:-1. However in the particular case of the Ecalle-Voronin modulus of the holonomy of a saddle-node then the map $\psi^{\infty}$ is always an affine map (a translation if we choose properly the coordinates on the spheres). In [8] we had shown that it was the only diffeomorphism which could be allowed in order to respect the properties of the node, namely to be linearizable as soon as resonant. Here we will give a more geometric explanation, using that the affine maps are the only global diffeomorphims of the sphere which have a fixed point at $\infty$.

4.1 Proposition The germ of diffeomorphism $\psi^{\infty}$ of the Martinet-Ramis modulus of a saddle-node is an affine map (a translation if we scale $\psi^{\infty \prime}(\infty)=1$ ).

Proof. We suppose that the vector field is defined in a neighborhood $U \times W$ with $U=\{x ;|x|<r\}$ and $W=\{y ;|y|<2\}$. We show below that all orbits of a vector field (2.1) starting in a neighborhood of the origin of the form $U_{1} \times W_{1}$ with $U_{1}=\left\{x ;|x|<r_{1}\right\}$ and $W_{1}=\{y ;|y| \leq 1\}$ intersect $|y|=1$, except possibly the center manifold. We want to study the correspondence between the points $(x, y)$ of $U_{1} \times W_{1}$ and the orbits of the holonomy map. Given a point $\left(x_{0}, y_{0}\right) \in U \times W$ with $\Re x_{0}<0$ we follow the trajectory through 
$\left(x_{0}, y_{0}\right)$. Its projection on the $x$-axis is included in a trajectory of $x^{2} \frac{\partial}{\partial x}$ tending to the origin tangentially to $R^{-}$. Similarly with a point where $\Re x_{0}>0,\left|x_{0}\right|<r / 2$ and $\left|\arg x_{0}\right|>\eta_{0}$ where $\eta_{0} \in(0, \pi / 4)$. If we now start with a point $\left(x_{0}, y_{0}\right)$ such that $\left|x_{0}\right|<r_{1}$, with $r_{1}>0$ sufficiently small and $\left|\arg x_{0}\right|<2 \eta_{0}$ then there exist $\theta_{1} \in(0, \pi / 2)$ and $\theta_{2} \in(-\pi / 2,0)$ such that the projection of the trajectory of $\left(x_{0}, y_{0}\right)$ for $v_{0}\left(\theta_{1}\right)=e^{i \theta_{1}} v_{0}$, call it $\gamma_{1}$, (resp. $v_{0}\left(\theta_{2}\right)=e^{i \theta_{2}} v_{0}$, call it $\gamma_{2}$, ) onto the $x$-axis in included in a trajectory of $e^{i \theta_{1}} x^{2} \frac{\partial}{\partial x}$ (resp. $e^{i \theta_{2}} x^{2} \frac{\partial}{\partial x}$ ) tending to the origin in the region $\Re x<0$.

Except possibly if we are on the center manifold the trajectory through $\left(x_{0}, y_{0}\right)$ will enter the cone $K_{2}$ and cut the cylinder $|y|=1$ at a value $y_{1}=e^{i \theta_{3}}, \theta_{3} \in(0,2 \pi]$. Following the curve $\left(x(\theta), y_{1} e^{i \theta}\right)$ contained in the leaf for $\theta \in\left[\theta_{3}, 2 \pi\right]$ (as in the construction of the holonomy map) allows to find where the leaf through $\left(x_{0}, y_{0}\right)$ cuts the section $y=1$. For a given $x_{0}$, the smaller $y_{0}$, the closer to $-\sqrt{\epsilon}$ the trajectory of $\left(x_{0}, y_{0}\right)$ intersects $|y|=1$.

The curves $\gamma_{1}$ and $\gamma_{2}$ are not homotopic in $U_{1} \backslash\{0\}$ and the procedure gives a surjection between $\left\{(x, y) ;|x|<r_{1},|\arg x|<2 \eta_{0},|y|<1\right\}$ and each of the fundamental domains of Figure 4(a). Hence the

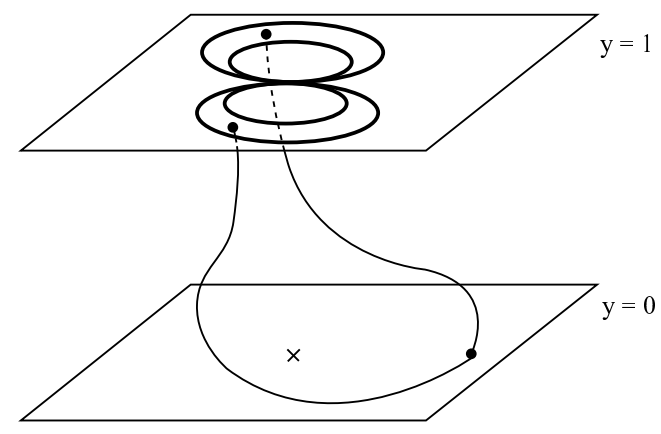

(a) $\epsilon=0$



(b) $\hat{\epsilon} \neq 0$

Figure 4: Construction of the first integral

map $\psi^{\infty}$ identifying orbits in each fundamental domain must be a global diffeomorphism with a fixed point at $\infty$ : it is then affine, and a translation for a good choice of coordinates on the spheres: $\psi^{\infty}(w)=w+C$. We now recover the geometric interpretation of $C$ : if $C=0$ the center manifold is not ramified hence analytic, while if $C \neq 0$, because of the ramifications the center manifold will intersect $|y|=1$.

\subsection{Unfolding of the Ecalle-Voronin modulus of the holonomy map of a saddle-node}

The following theorem was shown in [8].

4.2 Theorem [8] A complete modulus of analytic classification of a germ of generic 1-parameter analytic family of diffeomorphisms unfolding a germ of diffeomorphism with a parabolic fixed point at the origin

$$
f_{\epsilon}(x)=x+\left(x^{2}-\epsilon\right)(1+h(x, \epsilon))
$$

with $h(x, \epsilon)=O(x, \epsilon)$ in prepared form (i.e. the parameter $\epsilon$ is an analytic invariant) is given by the family of unfoldings $\left(\psi_{\hat{\epsilon}}^{0}, \psi_{\hat{\epsilon}}^{\infty}\right)_{\hat{\epsilon} \in \tilde{V}}$ of the Ecalle-Voronin modulus, together with a(0), where $\psi_{\hat{\epsilon}}^{0}$, $\psi_{\hat{\epsilon}}^{\infty}$ are germs of analytic diffeomorphisms depending analytically of $\hat{\epsilon}$ for $\hat{\epsilon} \neq 0$ and continuously on $\hat{\epsilon}$ near $\epsilon=0$, and where $\hat{\epsilon}$ is in a sector $\tilde{V}$ of the universal covering of $\epsilon$-space given by $\arg \hat{\epsilon} \in(-\pi+\delta, 3 \pi-\delta),|\hat{\epsilon}|<\rho(\delta)$, where $\delta>0$ can be chosen arbitrarily small and $\rho(\delta)>0$ depends on $\delta$ (and of the family).

We now show that for the particular case of the holonomy map of the unfolding of a saddle-node, not only $\psi^{\infty}$ is an affine map for $\epsilon=0$, but its unfolding $\psi_{\hat{\epsilon}}^{\infty}$ remains an affine map for $\hat{\epsilon} \neq 0$. As before the geometric idea is that the map $\psi_{\hat{\epsilon}}^{\infty}$ must be global. 
4.3 Remark We need to consider the variable $\bar{x}$ of (4.2) and the corresponding formal invariant $\bar{a}$ if we want to apply Theorem 4.2 verbatim.

4.4 Theorem We consider a family (2.3) defined in a neighborhood of the origin of the form $U \times W$ where $W=\{y ;|y|<2\}$ and the holonomy of the unfolded system (which is a generic unfolding of the holonomy map of the strong separatrix) whose modulus of analytic classification is given by the family $\left(\psi_{\hat{\epsilon}}^{0}, \psi_{\hat{\epsilon}}^{\infty}\right)_{\hat{\epsilon} \in V}$ unfolding the Ecalle-Voronin modulus of the holonomy map for $\epsilon=0$. Then the map $\psi_{\hat{\epsilon}}^{\infty}$ is an affine map (a translation $w \mapsto w+C(\hat{\epsilon})$ for adequate coordinates on the spheres).

Proof. As in Proposition 4.1 we will show that the map $\psi_{\hat{\epsilon}}^{\infty}$ is a global diffeomorphism fixing $\infty$. As $\psi_{\hat{\epsilon}}^{\infty}$ depends analytically on $\hat{\epsilon}$ it suffices to prove this property for $\hat{\epsilon}$ in a sector of small opening, for instance $\arg \hat{\epsilon} \in(-\delta, \delta)$ with $\delta>0$ small. In this case the $x$-eigenvalue of $-\sqrt{\epsilon}$ (resp. $\sqrt{\epsilon}$ ) has a negative (resp. positive) real part. As before we associate to points $\left(x_{0}, y_{0}\right)$ with $\left|x_{0}\right|<r / 2,\left|y_{0}\right|<1$, points of the section $y=1$ which belong to the same leaf. This is done by associating to $\left(x_{0}, y_{0}\right)$ a point of its leaf located on the cylinder $|y|=1$ and pushing the construction of the holonomy map. As in Proposition 4.1 we consider points $x_{0}$ such that $\left.\left|\arg \left(x_{0}-\sqrt{\epsilon}\right)\right|<\pi / 4\right\}$. There exists $\theta_{1} \in(0, \pi / 4)$ and $\theta_{2} \in(-\pi / 4,0)$ such that the trajectory of the 1-dimensional system $d x / d t=e^{i \theta_{j}}\left(x^{2}-\epsilon\right)$ through $x_{0}$ with $\left|x_{0}\right|<r_{1}<r$ tends to $-\sqrt{\epsilon}$ winding around $\sqrt{\epsilon}$ in the positive (resp. negative) direction for $j=1$ (resp. $j=2$ ), see Figure 4(b) and Figure 5. Moreover the $y$-eigenvalues of $\pm \sqrt{\epsilon}$ both have positive real parts. Hence trajectories of the

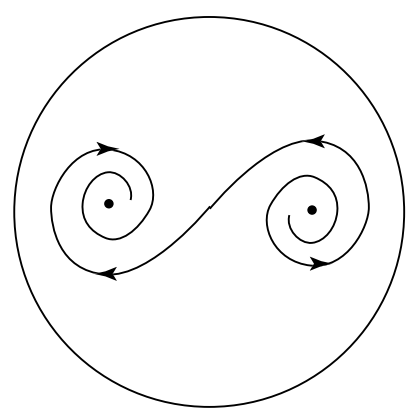

(a) $v_{\epsilon}\left(\theta_{1}\right), \theta_{1}>0$

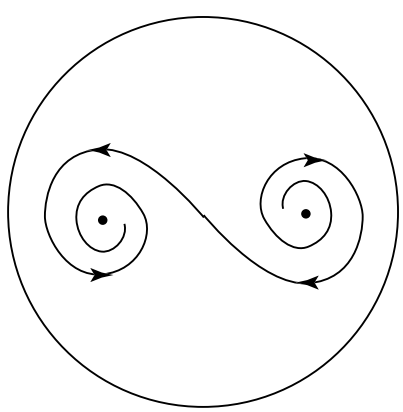

(b) $v_{\epsilon}\left(\theta_{2}\right), \theta_{2}<0$

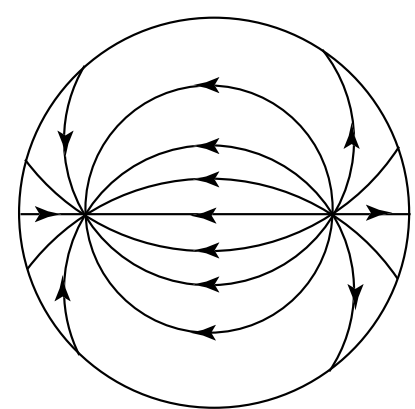

(c) $v_{\epsilon}$ for $\epsilon>0$

Figure 5: The projection of trajectories of $v_{\epsilon}(\theta)$ on the $x$-axis

corresponding vector fields $v_{\epsilon}\left(\theta_{j}\right)$ through $\left(x_{0}, y_{0}\right)$ will intersect $|y|=1$ except possibly if $\left(x_{0}, y_{0}\right)$ is on the graph of the unfolding of the center manifold described in Theorem 3.1. Moreover all points of one fundamental neighborhood (resp. the other) for the holonomy map lie on trajectories of points $\left(x_{0}, y_{0}\right)$ for the vector field $v_{\epsilon}\left(\theta_{1}\right)$ for some $\theta_{1}>0\left(\right.$ resp. $v_{\epsilon}\left(\theta_{2}\right)$ for some $\left.\theta_{2}<0\right)$. Hence the transition map $\psi_{\hat{\epsilon}}^{\infty}$ is global.

\subsection{Comparing the family (2.3) to the family model}

The family model for the unfolding of a saddle node is given by

$$
\begin{aligned}
\dot{x} & =x^{2}-\epsilon \\
\dot{Y} & =Y(1+a(\epsilon) x) .
\end{aligned}
$$

For simplicity we will write $a$ instead of $a(\epsilon)$. Its first integral is

$$
\tilde{H}_{\epsilon}(x, Y)=Y\left(x^{2}-\epsilon\right)^{-\frac{a}{2}}\left(\frac{x+\sqrt{\epsilon}}{x-\sqrt{\epsilon}}\right)^{\frac{1}{2 \sqrt{\epsilon}}}=Y F(x) .
$$


The integral is in general ramified at $x= \pm \sqrt{\epsilon}$.

The deformation in the Glutsyuk point of view. The point of view of Glutsyuk described in [2] and valid for a cone in $\epsilon$-space avoiding the Siegel direction is that deformations $H_{\epsilon}^{+}$and $H_{\epsilon}^{-}$of the first integrals exist in neighborhoods of the singular points $( \pm \sqrt{\epsilon}, 0)$ with the following properties:

- They are the "canonical integrals" of the form $\left(x_{1}-\sqrt{\epsilon}\right)^{\frac{1}{\mu^{+}}} y_{1}$ and $\left(x_{1}+\sqrt{\epsilon}\right)^{\frac{1}{\mu^{-}}} y_{1}$ where $\left(x_{1}, y_{1}\right.$ are linearizing coordinates at $( \pm \sqrt{\epsilon}, 0)$, so that $x_{1}= \pm \sqrt{\epsilon}$ and $y_{1}=0$ are the analytic separatrices of the singular points and $\mu^{ \pm}$are the quotients of eigenvalues at $\pm \sqrt{\epsilon}$.

- $H_{\epsilon}^{+}$(resp. $H_{\epsilon}^{-}$) tends to $H_{0}^{+}$(resp. $\left.H_{0}^{-}\right)$as $\epsilon \rightarrow 0$.

- The transition between $H_{\epsilon}^{+}$and $H_{\epsilon}^{-}$tends to the Martinet-Ramis modulus (the domain becomes disconnected at the limit).

We will not give more details on the Glutsyuk point of view and will concentrate on the "Lavaurs point of view", which consists in comparing the family (2.3) to the model family (4.6) "between the singular points" and to read the incompatibility of a full comparison when turning around the singular points.

\section{The deformation in the Lavaurs point of view.}

4.5 Theorem We consider a prepared family (2.3). Then for $\hat{\epsilon} \in V$ there exists a change of coordinate $Y=Y(x, y, \epsilon)$, holomorphic in $y$ and in $x \neq \pm \sqrt{\epsilon}$, and ramified in $x$ at $\pm \sqrt{\epsilon}$ (see Figure $6 a)$ ) bringing the system (2.3) to the model family (4.6). The change of coordinate is holomorphic in $\hat{\epsilon}$ for $\hat{\epsilon} \neq 0$ and continuous in $\hat{\epsilon}$ near $\hat{\epsilon}=0$. Moreover near $\pm(\sqrt{\epsilon}, 0) Y(x, y, \epsilon)$ has an asymptotic expansion $Y(x, y, \epsilon)=$ $y+\sum_{i=0}^{\infty} b_{i, \epsilon}(x) y^{i}$ where $\lim _{x \rightarrow \pm \sqrt{\epsilon}} b_{i, \epsilon}(x)=0$.

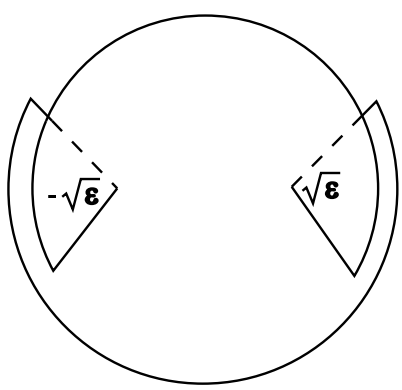

(a) The domain in $x$ space



(b) The Lavaurs map and transition maps

Figure 6: The domain in $x$-ccordinate

Proof. The proof goes as follows. We define two first integrals for the system in the neighborhood of the origin, one for each fundamental domain of the holonomy map on $\{y=1\}$. On each point of a fundamental domain the first integral is the spherical coordinate of that point for the holonomy map. Each first integral can be continued (in a ramified way) on all $U \times\{y=1\}$ by asking that it be constant on orbits of the holonomy map. For $\epsilon \neq 0$ the two first integrals can be compared by means of the global transition map between the two fundamental domains. In the spherical coordinates this map is a linear map $L$, the Lavaurs map (see Figure 6b)). So, instead of working with two first integrals, we work with one first integral $H(x, y, \epsilon)$ built with one of the fundamental domains. This first integral is ramified for $x= \pm \sqrt{\epsilon}$. The transition from one branch to the other can be seen through the maps $\psi_{\hat{\epsilon}}^{0} \circ L$ when turning 
around $-\sqrt{\epsilon}$ (resp. $\psi_{\hat{\epsilon}}^{\infty} \circ L$ when turning around $\sqrt{\epsilon}$ ). Comparing to the first integral (4.7) of the model this suggests the change of coordinate

$$
Y(x, y, \epsilon)=\frac{H(x, y, \epsilon)}{F(x)} .
$$

To finish the proof we first need to extend the construction of the first integral to a neighborhood $U \times W_{1}$. We also have to check that we can extend the proof of the construction of the first integral done only for a small sector in $\hat{\epsilon}$-space in Theorem 4.4 to the full sector $V$. We then have to check that $H(x, y, \epsilon)$ has the required asymptotic expansion allowing the conclusions of the theorem for the asymptotic expansion of $Y(x, y, \epsilon)$.

We first extend the first integral $H$ to $\{|y|=1\}$ : this is done as in the proof of Proposition 4.1. To extend it to $\{|y|<1\}$ we reproduce verbatim the construction of the first integral in the proof of Theorem 4.4 (see also Figure $4 \mathrm{~b})$ ). Indeed as $\hat{\epsilon} \in V$ it is always possible to choose $\theta_{1} \in(0, \pi / 2)$ or $\theta_{2} \in(-\pi / 2,0)$ such that $e^{i \theta_{1}} v_{\epsilon}$ or $e^{i \theta_{2}} v_{\epsilon}$ have singular points with eigenvalues in $y$ with positive real part and such that the eigenvalue in $x$ has negative (resp. positive) real part at $x=-\sqrt{\epsilon}$ (resp. $x=\sqrt{\epsilon}$ ). In particular we get that any of the fundamental domains is completely covered by points belonging to orbits starting in the neighborhood of $(\sqrt{\epsilon}, 0)$.

We let $H(x, y, \epsilon)=0$ for $y=g_{\hat{\epsilon}}(x)$. The $H$ obtained in this way is obviously analytic in $y$ and ramified in $x$ at $x= \pm \sqrt{\epsilon}$.

We must now study the asymptotic properties of $H$ near $(x, y)=( \pm \sqrt{\epsilon}, 0)$. For $\epsilon=0$ it corresponds to a first integral (4.7) constructed by means of a change of coordinate $y \mapsto Y(y, x, \epsilon)$ bringing the system (2.3) to the family model (4.6).

Near $\sqrt{\epsilon}$ we compare our integral with a standard integral of the form

$$
H_{1}\left(x, y_{1}, \epsilon\right)=\frac{y_{1}-k_{\hat{\epsilon}}(x)}{(x-\sqrt{\epsilon})^{\frac{1+a \sqrt{\epsilon}}{2 \sqrt{\epsilon}}}}
$$

where $y \mapsto y_{1}$ is a change of coordinate bringing the node to normal form and $y_{1}-k_{\hat{\epsilon}}(x)=0$ is the trajectory $y-g_{\hat{\epsilon}}(x)=0$ through $\sqrt{\epsilon}$ written in the coordinate $y_{1}$ and hence possibly ramified at $x=\sqrt{\epsilon}$. We get a bijection between the leaves of the foliation in the neighborhood of the node and the values of $H$ on one side, the values of $H_{1}$ on the other side. The map transforming $H$ to $H_{1}$ is a global diffeomorphism of $C P^{1}$ preserving 0 and $\infty$ : it is hence a linear map. Then $H(x, y, \epsilon)=C(\epsilon) H_{1}\left(x, y_{1}(x, y, \epsilon), \epsilon\right)$ with $C(\epsilon) \neq 0$, yielding that

$$
Y(x, y, \epsilon)=\frac{H(x, y, \epsilon)}{F(x)}=C(\epsilon)\left(y_{1}(x, y, \epsilon)-k_{\hat{\epsilon}}(x)\right)
$$

in the neighborhood of $\sqrt{\epsilon}$. Hence $Y(x, y, \epsilon)$ vanishes at $x=\sqrt{\epsilon}$ and has the desired asymptotic properties.

Near $-\sqrt{\epsilon}$ we could not find such an elegant argument. Our proof is more analytic. In this case the first integral takes small values. We must show that it remains small when divided by $m_{\hat{\epsilon}}(x)$. For that we use that $H(x+\sqrt{\epsilon})^{-\frac{1-a \sqrt{\epsilon}}{2 \sqrt{\epsilon}}}$ is bounded in the neighborhood of $(x, y)=(-\sqrt{\epsilon}, 0)$. This comes from the construction of the spherical coordinates. Indeed the first derivative of the holonomy map at $-\sqrt{\epsilon}$ is given by $\exp \left(-\frac{2 \sqrt{\epsilon}}{1-a \sqrt{\epsilon}}\right)$. When building the spherical coordinates the point $-\sqrt{\epsilon}$ is sent to the origin and the holonomy restricted to the sphere becomes the identity. A ramification $x \mapsto(x+\sqrt{\epsilon})^{-\frac{2 \sqrt{\epsilon}}{1-a \sqrt{\epsilon}}}+\ldots$ is necessary to achieve this. The rest follows by remarking that the first integral $H(x, y, \epsilon)$ vanishes along $y=g_{\hat{\epsilon}}(x)$. 


\section{Complete invariant of orbital analytic classification for a generic fam- ily of vector fields unfolding a generic saddle-node}

5.1 Definition Two germs of analytic families of vector fields, $v_{\epsilon_{1}}\left(x_{1}, y_{1}\right)$ (resp. $w_{\epsilon_{2}}\left(x_{2}, y_{2}\right)$ ) unfolding a saddle-node at the origin for $\epsilon_{1}=0$ (resp. $\epsilon_{2}=0$ ) are weakly orbitally equivalent if there exists a germ of map $K=(h, \Phi, k),\left(\epsilon_{1}, x_{1}, y_{1}\right) \mapsto\left(h\left(\epsilon_{1}\right), \Phi\left(\epsilon_{1}, x_{1}, y_{1}\right), k\left(\epsilon_{1}, x_{1}, y_{1}\right)\right)$ fibered over the parameter space where

i) $h: \epsilon_{1} \mapsto \epsilon_{2}=h\left(\epsilon_{1}\right)$ is a germ of homeomorphism preserving the origin.

ii) There exists a representative $\Phi_{\epsilon_{1}}\left(x_{1}, y_{1}\right)=\Phi\left(\epsilon_{1}, x_{1}, y_{1}\right)$ which is an analytic diffeomorphism in $\left(x_{1}, y_{1}\right)$ on a small neighborhood of the origin in $\left(x_{1}, y_{1}\right)$-space, for $\epsilon_{1}$ in a sufficiently small neighborhood of the origin.

iii) There exists a representative $k_{\epsilon_{1}}\left(x_{1}, y_{1}\right)=k\left(\epsilon_{1}, x_{1}, y_{1}\right)$ depending analytically of $\left(x_{1}, y_{1}\right)$ for $\left(x_{1}, y_{1}\right)$ in a small neighborhood of the origin in $\left(x_{1}, y_{1}\right)$-space with values in $C$, and non-vanishing in a neighborhood of the origin for $\epsilon_{1}$ in a sufficiently small neighborhood of the origin.

iv) The change of coordinates $\Phi_{\epsilon_{1}}$ and the scaling of time $k_{\epsilon_{1}}$ is an equivalence between $v_{\epsilon_{1}}\left(x_{1}, y_{1}\right)$ and $w_{h\left(\epsilon_{1}\right)}\left(x_{2}, y_{2}\right)$ over a ball of small radius $r>0$ :

$$
w_{h\left(\epsilon_{1}\right)}\left(\Phi_{\epsilon_{1}}\left(x_{1}, y_{1}\right)\right)=k\left(\epsilon_{1}, x_{1}, y_{1}\right)\left(\Phi_{\epsilon_{1}}\right)_{*}\left(v_{\epsilon_{1}}\left(x_{1}, y_{1}\right)\right)
$$

5.2 Theorem We consider a family unfolding a generic saddle-node and its prenormal form (2.3). The modulus of analytic classification of the holonomy map of the unfolded vector field, namely the family $\left(\psi_{\epsilon}^{0}, \psi_{\epsilon}^{\infty}\right)_{\hat{\epsilon} \in V}$, together with a $(0)$ is a complete modulus of orbital analytic classification under weak orbital equivalence.

Proof. We consider two families of the form (2.3) with respective coordinates $\left(x_{1}, y_{1}\right)$ and $\left(x_{2}, y_{2}\right)$

$$
\begin{aligned}
& \dot{x}_{1}=x_{1}^{2}-\epsilon_{1} \\
& \dot{y}_{1}=\left(x_{1}^{2}-\epsilon_{1}\right) f_{0}\left(x_{1}\right)+y_{1}\left(1+a_{1}\left(\epsilon_{1}\right) x_{1}\right)+o\left(y_{1}\right),
\end{aligned}
$$

and

$$
\begin{aligned}
& \dot{x}_{2}=x_{2}^{2}-\epsilon_{2} \\
& \dot{y}_{2}=\left(x_{2}^{2}-\epsilon_{2}\right) g_{0}\left(x_{2}\right)+y_{2}\left(1+a_{2}\left(\epsilon_{2}\right) x_{2}\right)+o\left(y_{2}\right) .
\end{aligned}
$$

such that $a_{1}(0)=a_{2}(0)$. As the parameter is an analytic invariant we can suppose that $\epsilon_{1}=\epsilon_{2}=\epsilon$. Moreover we must show that there exists a change of coordinate transforming the first system for a value of $\epsilon$ to the second system for the same value of $\epsilon$. By choosing the coordinates appropriately on the spheres we can suppose that the two families have the same invariants $\left(\psi_{\hat{\epsilon}}^{0}, \psi_{\hat{\epsilon}}^{\infty}\right)_{\hat{\epsilon} \in V}$ valid in a common neighborhood. We fix $\hat{\epsilon} \in V$ : this allows not to write the dependence in $\hat{\epsilon}$. We construct changes of coordinates $\phi_{1}\left(x_{1}, y_{1}\right)=$ $\left(x_{1}, Y_{1}\left(x_{1}, y_{1}, \epsilon\right)\right)$ (resp. $\phi_{2}\left(x_{2}, y_{2}\right)=\left(x_{2}, Y_{2}\left(x_{2}, y_{2}, \epsilon\right)\right)$ ) (depending on $\left.\hat{\epsilon}\right)$ transforming (5.2) (resp. (5.3)) into the family model (4.6) as in Theorem 4.5. These changes of coordinates are ramified at $\pm \sqrt{\epsilon}$. Then the change of coordinate $\Phi=\phi_{2}^{-1} \circ \phi_{1}$ is analytic in a neighborhood $U \times W$ and transforms the first system into the second. Indeed if we call $L$ the Lavaurs map (see proof of Theorem 4.5) and we call $H_{j}^{+}, Y_{j}^{+}$and $\phi_{j}^{+}$(resp. $H_{j}^{-}, Y_{j}^{-}$and $\phi_{j}^{-}$) the branches of $H_{j}, Y_{j}, \phi_{j}$ obtained by turning around $-\sqrt{\epsilon}$ in the positive (resp. negative direction) then $H_{j}^{+}=L \circ \psi_{\hat{\epsilon}}^{0}\left(H_{j}^{-}\right)$, yielding

$$
Y_{j}^{+}=\frac{1}{F(x)} L \circ \psi_{\hat{\epsilon}}^{0}\left(F(x) Y_{j}^{-}\right) .
$$

Similarly when turning around $+\sqrt{\epsilon}$. Hence $\left(\phi_{2}^{+}\right)^{-1} \circ \phi_{1}^{+}=\left(\phi_{2}^{-}\right)^{-1} \circ \phi_{1}^{-}$, i.e. $\Phi$ is analytic and not ramified.

An equivalent of Theorem 3.2 holds for the saddle. 
5.3 Theorem We consider a family unfolding a generic saddle-node, its prenormal form (2.3) and its modulus of analytic classification $\left(\psi^{0}, \psi^{\infty}\right)$ as in Theorem 5.2. Let

$$
\psi^{0}(w)=w+\sum_{n \geq 2} b_{n} w^{n}
$$

Then $\forall p, q, k \in N$ with $1 \leq q<p$ there exist polynomials

$$
L_{p, q, k}^{\infty}\left(b_{2}, \ldots, b_{k p+1}\right)
$$

such that if $L_{p, q, k}^{\infty}\left(b_{2}, \ldots, b_{k p+1}\right) \neq 0$ then the saddle point $(-\sqrt{\epsilon}, 0)$ is non integrable of order $\leq k$ as soon as the ratio of its eigenvalues is of the form $-\frac{p}{n}$ with $n$ large and $n \equiv q(\bmod p)$. If $\psi^{0}$ is nonlinear, then at least one of the $L_{p, q, k}^{\infty}\left(b_{2}, \ldots, b_{k p+1}\right) \neq 0$.

Proof. The proof follows from the corresponding theorem for the holonomy map (see Theorem 8.1 of [8] and [9]): the polynomial $L_{p, q, k}$ is the $k$-th coefficient of the normal form of the resonant diffeomorphism $\exp (2 \pi i q / p) \psi^{0}(w)$.

We call this phenomenon parametric resurgence. Indeed the divergence of the normalizing transformation for a saddle-node coming from the nonlinearity of $\psi^{0}$ unfolds as an "incompatibility" in the system. This incompatibility is necessarily carried by the singular point of saddle type for discrete sequences of parameter values. Similarly the nonlinearity of $\psi^{\infty}$ induces a parametric resurgence phenomenon at the node (see Theorem 3.2).

\section{Study of an example: the Riccati equation}

\subsection{The general case}

We study the following family of Riccati equations

$$
\begin{aligned}
& \dot{x}=x^{2}-\epsilon \\
& \dot{y}=y(1+a(\epsilon) x)+f_{0}(x, \epsilon)\left(x^{2}-\epsilon\right)+f_{2}(x, \epsilon) y^{2} .
\end{aligned}
$$

6.1 Proposition There exists a change of coordinate

$$
Y=\frac{y}{1+B(\epsilon) y+C(\epsilon)(x-\sqrt{\epsilon}) y}
$$

depending analytically on $\epsilon$ belonging to the Riemann surface of $\sqrt{\epsilon}$ and transforming (6.1) into

$$
\begin{aligned}
\dot{x} & =x^{2}-\epsilon \\
\dot{Y} & =Y\left(1+a(\epsilon) x+O\left(x^{2}-\epsilon\right)\right)+\bar{f}_{0}(x)\left(x^{2}-\epsilon\right)+\bar{f}_{2}(x)\left(x^{2}-\epsilon\right) Y^{2} .
\end{aligned}
$$

A further change $X=X(x, \epsilon)$ brings the system to the form

$$
\begin{aligned}
\dot{X} & =X^{2}-\epsilon \\
\dot{Y} & =Y(1+\bar{a}(\epsilon) X)+\tilde{f}_{0}(X)\left(X^{2}-\epsilon\right)+\tilde{f}_{2}(X)\left(X^{2}-\epsilon\right) Y^{2}
\end{aligned}
$$

Proof. We let

$$
f_{2}(x, \epsilon)=\left(x^{2}-\epsilon\right) K(x, \epsilon)+c(\epsilon)(x-\sqrt{\epsilon})+b(\epsilon) .
$$

Replacing (6.2) into (6.1) and asking the transformed equation to have the form (6.3) yields to

$$
\left\{\begin{array}{l}
B(\epsilon)=\frac{b(\epsilon)}{1+a \sqrt{\epsilon}} \\
C(\epsilon)=\frac{c(\epsilon)-a B(\epsilon)}{1-a \sqrt{\epsilon}} .
\end{array}\right.
$$


The change in $X$ is done using Kostov's theorem as in Section 3.

Hence from now on we only study the particular family of Riccati equations

$$
\begin{aligned}
& \dot{x}=x^{2}-\epsilon \\
& \dot{y}=y(1+a x)+f_{0}(x)\left(x^{2}-\epsilon\right)+f_{2}(x)\left(x^{2}-\epsilon\right) y^{2},
\end{aligned}
$$

where $f_{0}, f_{2}$ are analytic functions of $x$ in a neighborhood of the origin. This equation has the two singular points $P_{ \pm}=( \pm \sqrt{\epsilon}, 0)$. These are well defined if we restrict $\epsilon$ to a sector $V$ of (3.2). The change of coordinate $Y=\frac{1}{y}$ transforms (6.6) into

$$
\begin{aligned}
& \dot{x}=x^{2}-\epsilon \\
& \dot{Y}=-Y(1+a x)-f_{2}(x)\left(x^{2}-\epsilon\right)-f_{0}(x)\left(x^{2}-\epsilon\right) Y^{2},
\end{aligned}
$$

which has the singular points $Q_{ \pm}$given by $(x, Y)=( \pm \sqrt{\epsilon}, 0)$. It is this change of coordinate which allows a complete calculation of the Martinet-Ramis modulus when $\epsilon=0$ ([6]).

There exists a neighborhood $U$ of the origin in $x$-space such that, if $\rho$ is sufficiently small, then $\pm \sqrt{\epsilon} \in U$. In the following we will put additional conditions on $U$.

The point $P_{-}$always has an analytic separatrix $y-g_{0}(x)=0$ (depending on $\hat{\epsilon}$ ) when $\hat{\epsilon}$ is in the sector $V$. This function $g_{0}(x)$ is the analytic solution of the differential equation

$$
\left(x^{2}-\epsilon\right) g_{0}^{\prime}(x)=g_{0}(x)(1+a x)+\left(x^{2}-\epsilon\right) f_{0}(x)+\left(x^{2}-\epsilon\right) g_{0}^{2}(x) f_{2}(x)
$$

satisfying $g_{0}(-\sqrt{\epsilon})=0$. From Theorem 3.1 the function $g_{0}(x)$ is defined for all $\hat{\epsilon} \in V$ as a multivalued function over the domain $U$ containing $\pm \sqrt{\epsilon}$, independent of $\epsilon$, and ramified at $\sqrt{\epsilon}$. For $\epsilon=0$ the graph of this function is the center manifold.

Similarly the point $Q_{+}$of system (6.7) always has an analytic separatrix of the form $Y-g_{2}(x)=0$ where $g_{2}(x)$ is a solution of the differential equation

$$
\left(x^{2}-\epsilon\right) g_{2}^{\prime}(x)+(1+a x) g_{2}(x)+\left(x^{2}-\epsilon\right) f_{2}(x)+\left(x^{2}-\epsilon\right) f_{0}(x) g_{2}^{2}(x)=0,
$$

with $g_{2}(\sqrt{\epsilon})=0$. As for $g_{0}$ the function $g_{2}(x)$ can be defined for all $\hat{\epsilon} \in V$ as a function on $U$ ramified at $-\sqrt{\epsilon}$

We construct a first integral of this equation by means of generalized Darboux factors and cofactors (see for instance [1]). Recall that a generalized Darboux factor is a function $G(x, y)$ such that

$$
\dot{G}(x, y)=\frac{\partial G}{\partial x} \dot{x}+\frac{\partial G}{\partial y} \dot{y}=G(x, y) K(x, y) .
$$

The function $K(x, y)$ is called the cofactor of $G(x, y)$. On purpose we do not define the classes of functions to which belong $F(x, y)$ and $K(x, y)$. Indeed the differential equation (6.8) (resp. (6.9)) satisfied by $g_{0}(x)$ (resp. $g_{2}(x)$ ) allows to consider $y-g_{0}(x)$ (resp. $\left.1-y g_{2}(x)\right)$ as a Darboux factor with cofactor given in (6.11) below.

The system (6.6) has the following generalized Darboux factors and cofactors (we simply write $a$ for $a(\epsilon))$ :

$$
\begin{array}{lc}
F_{1}(x)=x-\sqrt{\epsilon} & K_{1}(x, y)=x+\sqrt{\epsilon} \\
F_{2}(x)=x+\sqrt{\epsilon} & K_{2}(x, y)=x-\sqrt{\epsilon} \\
F_{3}(x, y)=y-g_{0}(x) & K_{3}(x, y)=\left(x^{2}-\epsilon\right) f_{2}(x) y+1+a x \\
& \quad+\left(x^{2}-\epsilon\right) f_{2}(x) g_{0}(x) \\
F_{4}(x, y)=1-g_{2}(x) y & K_{4}(x, y)=\left(x^{2}-\epsilon\right) f_{2}(x) y \\
& -\left(x^{2}-\epsilon\right) f_{0}(x) g_{2}(x) \\
F_{5}(x, y)=\exp \left(-\int_{-\sqrt{\epsilon}}^{x} f_{2}(\xi) g_{0}(\xi) d \xi\right) & K_{5}(x)=-\left(x^{2}-\epsilon\right) f_{2}(x) g_{0}(x) \\
F_{6}(x, y)=\exp \left(-\int_{\sqrt{\epsilon}}^{x} f_{0}(\xi) g_{2}(\xi) d \xi\right) & K_{6}(x)=-\left(x^{2}-\epsilon\right) f_{0}(x) g_{2}(x)
\end{array}
$$


As there exists a linear combination $\sum_{i=1}^{6} \beta_{i} K_{i}=0$, this yields a first integral $H_{\epsilon}(x, y)=\prod_{i=1}^{6} F_{i}^{\beta_{i}}$, i.e.

$$
H_{\epsilon}(x, y)=\left(x^{2}-\epsilon\right)^{-\frac{a}{2}}\left(\frac{x+\sqrt{\epsilon}}{x-\sqrt{\epsilon}}\right)^{\frac{1}{2 \sqrt{\epsilon}}} F_{5}(x) F_{6}(x) \frac{y-g_{0}(x)}{1-y g_{2}(x)} .
$$

where $F_{5}(x), F_{6}(x)=1+O(x)$.

For each $\epsilon$ the first integral is defined on a domain $U \times W$ where $W$ is a small disk in $y$-space. The first integral is ramified at $\pm \sqrt{\epsilon}$ if we can consider it as a univalued function defined on $\hat{U} \times W$ where $\hat{U}$ is a domain as in Figure 6a).

When turning around $\pm \sqrt{\epsilon}$ this yields two integrals $H_{1, \pm, \epsilon}$ and $H_{2, \pm, \epsilon}$ depending whether we turn around $\pm \sqrt{\epsilon}$ in the positive (resp. negative) direction.

\subsection{Proposition (1) $H_{2,+, \epsilon}=A(\epsilon) H_{1,+, \epsilon}+B(\epsilon)$,}

(2) $H_{2,-, \epsilon}=\frac{C(\epsilon) H 1,-, \epsilon}{1+D(\epsilon) H_{1,-, \epsilon}}$

where $A(\epsilon), C(\epsilon) \neq 0$ and $B(\epsilon), D(\epsilon)$ depend continuously on $\hat{\epsilon} \in V$.

Proof. The functions $g_{0}(x)$ and $F_{5}(x)$ are ramified at $x=\sqrt{\epsilon}$ while the functions $g_{2}(x)$ and $F_{6}(x)$ are ramified at $-\sqrt{\epsilon}$. Also the map

$$
F(x)=\left(x^{2}-\epsilon\right)^{-\frac{a}{2}}\left(\frac{x+\sqrt{\epsilon}}{x-\sqrt{\epsilon}}\right)^{\frac{1}{2 \sqrt{\epsilon}}}
$$

is ramified at the two points $\pm \sqrt{\epsilon}$. Let us call $\bar{g}_{0}, \bar{F}_{5}, \bar{F}$ (resp. $\hat{g}_{0}, \hat{F}_{5}, \hat{F}$ ) the extensions of $g_{0}, F_{5}, F$ when turning around $\sqrt{\epsilon}$ in the positive (resp. negative) direction. Then

$$
\begin{aligned}
H_{1,+, \epsilon} & =\bar{F}(x) \bar{F}_{5}(x) F_{6}(x) \frac{y-\bar{g}_{0}(x)}{1-y g_{2}(x)} \\
H_{2,+, \epsilon} & =\hat{F}(x) \hat{F}_{5}(x) F_{6}(x) \frac{y-\hat{g}_{0}(x)}{1-y g_{2}(x)}
\end{aligned}
$$

We eliminate $y$ between the two equations of (6.14), yielding

$$
H_{2,+, \epsilon}=\frac{\hat{F}(x) \hat{F}_{5}(x)\left(1-g_{2}(x) \hat{g}_{0}(x)\right)}{\bar{F}(x) \bar{F}_{5}(x)\left(1-g_{2}(x) \bar{g}_{0}(x)\right)} H_{1,+, \epsilon}+\frac{\hat{F}(x) \hat{F}_{5}(x) F_{6}(x)\left(\bar{g}_{0}(x)-\hat{g}_{0}(x)\right)}{1-g_{2}(x) \bar{g}_{0}(x)} .
$$

Similarly let us call $\tilde{g}_{2}, \tilde{F}_{6}, \tilde{F}$ (resp. $\check{g}_{2}, \check{F}_{6}, \check{F}$ ) the extensions of $g_{2}, F_{6}, F$ when turning around $-\sqrt{\epsilon}$ in the positive (resp. negative) direction. Then

$$
\begin{aligned}
& H_{1,-, \epsilon}=\tilde{F}(x) F_{5}(x) \tilde{F}_{6}(x) \frac{y-g_{0}(x)}{1-y \tilde{g}_{2}(x)} \\
& H_{2,-, \epsilon}=\tilde{F}(x) F_{5}(x) \check{F}_{6}(x) \frac{y-g_{0}(x)}{1-y \check{g ̆ g}_{2}(x)} .
\end{aligned}
$$

We eliminate $y$ between the two equations of (6.16), yielding

$$
H_{2,-, \epsilon}=\frac{\check{F}(x) F_{5}(x) \check{F}_{6}(x)\left(1-\tilde{g}_{2}(x) g_{0}(x)\right) H_{1,-, \epsilon}}{\left(\tilde{g}_{2}(x)-\check{g}_{2}(x)\right) H_{1,-, \epsilon}+\tilde{F}(x) F_{5}(x) \tilde{F}_{6}(x)\left(1-g_{0}(x) \check{g}_{2}(x)\right)} .
$$

\subsection{The particular case of the linear equation}

We limit ourselves to the case of $f_{2} \equiv 0$ in (6.1). In this case $\psi_{\epsilon}^{0}$ is linear and $\psi_{\epsilon}^{\infty}(w)=w+C(\epsilon)$ for adequate choices of coordinates on the spheres. We calculate explicitly $C(\epsilon)$ in this case. Note that the general case can be reduced to this particular case when the function $g_{2}(x, \epsilon)$ is analytic. 
6.3 Theorem i) Let $f_{0}(x)=\sum_{n \geq 0} b_{n}(\epsilon)(x+\sqrt{\epsilon})^{n}$. Then the modulus has the form $\left(\psi_{\epsilon}^{0}, \psi_{\epsilon}^{\infty}\right)$ with $\psi_{\epsilon}^{0}$ linear and $\psi_{\epsilon}^{\infty}(w)=w+C(\epsilon)$ where

$$
C(\epsilon)=-2 i \pi \frac{\Gamma\left(\frac{1-a \sqrt{\epsilon}}{2 \sqrt{\epsilon}}\right)(2 \sqrt{\epsilon})^{-a}}{\Gamma\left(\frac{1+a \sqrt{\epsilon}}{2 \sqrt{\epsilon}}\right)} \sum_{n \geq 0} \frac{b_{n}(\epsilon)}{\Gamma(n-a+2)} \prod_{j=0}^{n}(2 j \sqrt{\epsilon}+1-a \sqrt{\epsilon}) .
$$

The function $C(\epsilon)$ is analytic in $\sqrt{\epsilon}$ near $\epsilon=0$ in the particular case $a(\epsilon) \equiv m$ with $m \in Z$. Otherwise it is analytic in $\sqrt{\epsilon}$ except at $\epsilon=0$ where it is a quotient of essential singularities at 0 . For $\epsilon$ going to 0 in the sectorial neighborhood $V$ the limit value is

$$
C(0)=-2 \pi i \sum_{n \geq 0} \frac{b_{n}(0)}{\Gamma(n-a+2)} .
$$

ii) The modulus space is isomorphic to $\mathcal{H}_{0}^{2}$ where $\mathcal{H}_{0}$ is the set of germs of holomorphic functions in $\sqrt{\epsilon}$ at the origin. Given $(a(\epsilon), d(\epsilon)) \in \mathcal{H}_{0}^{2}$ the modulus is given by

$$
\psi_{\epsilon}^{0}(w)=e^{-2 \pi i a(\epsilon)} w, \quad \psi_{\epsilon}^{\infty}(w)=w-2 i \pi \frac{\Gamma\left(\frac{1-a(\epsilon) \sqrt{\epsilon}}{2 \sqrt{\epsilon}}\right)(2 \sqrt{\epsilon})^{-a(\epsilon)}}{\Gamma\left(\frac{1+a(\epsilon) \sqrt{\epsilon}}{2 \sqrt{\epsilon}}\right)} d(\epsilon) .
$$

iii) Any family (6.1) with $f_{2} \equiv 0$ is weakly orbitally equivalent to a family of the form

$$
\begin{aligned}
& \dot{x}=x^{2}-\epsilon \\
& \dot{y}=y(1+a(\epsilon) x)+c_{N}(\epsilon) x^{N}
\end{aligned}
$$

with $N>|a(\epsilon)|$.

Proof. We know that the first integral has the form

$$
H_{\epsilon}(x, y)=\left(y-g_{0}(x)\right)\left(x^{2}-\epsilon\right)^{-\frac{a}{2}}\left(\frac{x+\sqrt{\epsilon}}{x-\sqrt{\epsilon}}\right)^{\frac{1}{2 \sqrt{\epsilon}}}=\left(y-g_{0}(x)\right) F(x),
$$

where $F(x)$ is given in (6.13). So we need only understand the behavior of the function $y=g_{0}(x)$ which is the analytic separatrix of $-\sqrt{\epsilon}$ (when this point is not a node). It has the form

$$
g_{0}(x)=(x+\sqrt{\epsilon})^{-\frac{1-a \sqrt{\epsilon}}{2 \sqrt{\epsilon}}}(x-\sqrt{\epsilon})^{\frac{1+a \sqrt{\epsilon}}{2 \sqrt{\epsilon}}} \int_{-\sqrt{\epsilon}}^{x} f_{0}(\zeta)(\zeta+\sqrt{\epsilon})^{\frac{1-a \sqrt{\epsilon}}{2 \sqrt{\epsilon}}}(\zeta-\sqrt{\epsilon})^{-\frac{1+a \sqrt{\epsilon}}{2 \sqrt{\epsilon}}} d \zeta .
$$

To calculate the integral part we make the change of coordinate $\xi=\frac{\zeta+\sqrt{\epsilon}}{2 \sqrt{\epsilon}}$ in the integral. Except for special values of $\epsilon$ for which $\sqrt{\epsilon}$ is a resonant node the function $g_{0}(x)$ generically has the form

$$
g_{0}(x)=(x+\sqrt{\epsilon})^{-\frac{1-a \sqrt{\epsilon}}{2 \sqrt{\epsilon}}}(x-\sqrt{\epsilon})^{\frac{1+a \sqrt{\epsilon}}{2 \sqrt{\epsilon}}}(A(\epsilon)+O(x-\sqrt{\epsilon}))
$$

in the neighborhood of $\sqrt{\epsilon}$. We calculate $A(\epsilon)$. It is given by

$$
\begin{aligned}
A(\epsilon) & =\sum_{n \geq 0} b_{n}(\epsilon)(2 \sqrt{\epsilon})^{n-a+1} e^{-\pi i \frac{1+a \sqrt{\epsilon}}{2 \sqrt{\epsilon}}} \int_{0}^{1} \xi^{n+\frac{1-a \sqrt{\epsilon}}{2 \sqrt{\epsilon}}}(1-\xi)^{-\frac{1+a \sqrt{\epsilon}}{2 \sqrt{\epsilon}}} d \xi \\
& =\sum_{n \geq 0} b_{n}(\epsilon)(2 \sqrt{\epsilon})^{n-a+1} e^{-\pi i \frac{1+a \sqrt{\epsilon}}{2 \sqrt{\epsilon}}} \frac{\Gamma\left(n+1+\frac{1-a \sqrt{\epsilon}}{2 \sqrt{\epsilon}}\right) \Gamma\left(1-\frac{1+a \sqrt{\epsilon}}{2 \sqrt{\epsilon}}\right)}{\Gamma(n-a+2)} .
\end{aligned}
$$

We must now see how the first integral $H$ of (6.21) ramifies when we turn around $\sqrt{\epsilon}$. Let us call $H_{1}$ the value of $h$ when we make one turn around $\sqrt{\epsilon}$. And let us treat the case when the node is not resonant as it is sufficient because of the analytic character of $C(\epsilon)$. (What happens when the node is resonant will be described below). Locally near $\sqrt{\epsilon}$ we have that $y-g_{0}(x)=y-h(x)-A(\epsilon) F^{-1}(x)$ where $h(x)$ is the 
analytic solution of (6.8) (which is here a linear equation) in the neighborhood of $\sqrt{\epsilon}$ and $-A(\epsilon) F^{-1}(x)$ is a solution of the homogeneous system. Hence the first integral has the form $H=(y-h(x)) F(x)-A(\epsilon)$. When we make one turn it becomes $H_{1}=B(\epsilon)(y-h(x)) F(x)-A(\epsilon)$, with

$$
B(\epsilon)=e^{-2 \pi i \frac{1+a \sqrt{\epsilon}}{2 \sqrt{\epsilon}}} .
$$

Hence

$$
H_{1}=B(\epsilon)(H+A(\epsilon))-A(\epsilon)=B(\epsilon)\left(H+A(\epsilon) \frac{B(\epsilon)-1}{B(\epsilon)}\right)=B(\epsilon)(H+C(\epsilon)),
$$

with $C(\epsilon)=A(\epsilon) \frac{B(\epsilon)-1}{B(\epsilon)}$. We finally get

$$
\begin{aligned}
C(\epsilon) & =-2 i \sum_{n \geq 0} b_{n}(\epsilon)(2 \sqrt{\epsilon})^{n-a+1} \sin \left(\pi \frac{1+a \sqrt{\epsilon}}{2 \sqrt{\epsilon}}\right) \frac{\Gamma\left(n+1+\frac{1-a \sqrt{\epsilon}}{2 \sqrt{\epsilon}}\right) \Gamma\left(1-\frac{1+a \sqrt{\epsilon}}{2 \sqrt{\epsilon}}\right)}{\Gamma(n-a+2)} \\
& =-2 \pi i \sum_{n \geq 0} \frac{b_{n}(\epsilon)(2 \sqrt{\epsilon})^{n-a+1}}{\Gamma(n-a+2)} \frac{\Gamma\left(n+1+\frac{1-a \sqrt{\epsilon}}{2 \sqrt{\epsilon}}\right)}{\Gamma\left(\frac{1+a \sqrt{\epsilon}}{2 \sqrt{\epsilon}}\right)},
\end{aligned}
$$

using that $\Gamma(z) \Gamma(1-z)=\frac{\pi}{\sin \pi z}$. The final form (6.18) follows by using $\Gamma(z+1)=z \Gamma(z)$.

In the particular case $a(\epsilon) \equiv m, m \in Z$ then the formula takes the simple form

$$
C(\epsilon)=-2 \pi i(2 \sqrt{\epsilon})^{-a} e(\epsilon) \sum_{n \geq 0} \frac{b_{n}(\epsilon)}{\Gamma(n-a+2)} \prod_{j=0}^{n}(2 j \sqrt{\epsilon}+1-m \sqrt{\epsilon}),
$$

where

$$
e(\epsilon)= \begin{cases}\prod_{j=m}^{-m-1}\left(\frac{1}{\sqrt{\epsilon}}+j\right) & m<0 \\ \prod_{j=-m}^{m-1} \frac{1}{\left(\frac{1}{\sqrt{\epsilon}}+j\right)} & m>0 \\ 1 & m=0 .\end{cases}
$$

In particular we see that $C(\epsilon)$ is analytic in $\sqrt{\epsilon}$ near $\epsilon=0$ when $a(\epsilon) \equiv m, m \in Z$.

In the limit when $\epsilon$ is very small we use

$$
\Gamma\left(\frac{1-a \sqrt{\epsilon}}{2 \sqrt{\epsilon}}\right) \sim\left(\frac{1}{2 \sqrt{\epsilon}}\right)^{-a} \Gamma\left(\frac{1+a \sqrt{\epsilon}}{2 \sqrt{\epsilon}}\right)
$$

to get the limit (6.19), which is the formula of Martinet-Ramis [6].

The function $1 / \Gamma$ has an essential singularity at infinity. The series in (6.18) converges, yielding that $C(\epsilon)$ is a uniform function multiplied by a quotient of two essential singularities at $\sqrt{\epsilon}=0$. It is accumulated by a sequence of zeroes (resp. a sequence of poles) located at the values of $\epsilon$ where $\frac{1+a \sqrt{\epsilon}}{2 \sqrt{\epsilon}}$ (resp. $\frac{1-a \sqrt{\epsilon}}{2 \sqrt{\epsilon}}$ ) is a negative integer $\leq-1$ (resp. $\leq-2$ ). The zeroes and poles annihilate each other when $a(\epsilon)=0$.

Part iii) follows from Theorem 5.2 and taking

$$
c_{N}(\epsilon)=\frac{\Gamma(N-a+2)}{\prod_{j=0}^{N}(2 j \sqrt{\epsilon}+1-a \sqrt{\epsilon})} \sum_{n \geq 0} \frac{b_{n}(\epsilon)}{\Gamma(n-a+2)} \prod_{j=0}^{n}(2 j \sqrt{\epsilon}+1-a \sqrt{\epsilon}) .
$$

Remark: The phenomemon near a resonant node with ratio of eigenvalues $n$ is interesting. The constant $B(\epsilon)-1$ vanishes as soon as the node is resonant since indeed no solutions are ramified in the case of a linearizable resonant node. To understand what happens at a resonant node, i.e. when $\frac{1+a \sqrt{\epsilon}}{2 \sqrt{\epsilon}}=n$, we let $\alpha=\frac{1+a \sqrt{\epsilon}}{2 \sqrt{\epsilon}}-n$ and look at what happens when $\alpha \rightarrow 0$. As the system is close to a resonant node, i.e. of the form $\left.x \frac{\partial}{\partial x}+\left[(n+\alpha) y+\beta x^{n}\right)\right] \frac{\partial}{\partial y}$, if beta $\neq 0$ then the function $h(x)$ contains a term in $x^{n}$ with a very 
large coefficient $A(\epsilon)$. If $C(\epsilon) \neq 0$ then $g_{0}(x)$ contains a ramified coefficient of the form $-A(\epsilon)(x-\sqrt{\epsilon})^{n}$. The sum of these two terms is of the form $\alpha A(\epsilon)(x-\sqrt{\epsilon})^{n} \omega(x-\sqrt{\epsilon}, \alpha)$, where

$$
\omega(x-\sqrt{\epsilon}, \alpha)= \begin{cases}\frac{(x-\sqrt{\epsilon})^{-\alpha}-1}{\alpha} & \alpha \neq 0 \\ -\ln (x-\sqrt{\epsilon}) & \alpha=0 .\end{cases}
$$

We know that when $C(0) \neq 0$, then $C(\epsilon) \neq 0$ for $\epsilon$ sufficiently small, yielding that $A(\epsilon)$ becomes infinite at all resonant nodes. However as $A(\epsilon)$ has a simple pole when $\alpha=0$ the limit of $\alpha A(\epsilon)$ exists when $\alpha \rightarrow 0$.

\section{Directions for further research}

We mention two natural directions for further research:

(1) The first is to identify precisely the modulus space for analytic families of vector fields unfolding a saddle-node.

(2) The second is to generalize the previous results for a saddle-node of arbitrary codimension.

\section{Acknowledgements}

The author thanks P. Elizarov, Y. Ilyashenko, P. Mardešić and R. Roussarie for helpful discussions.

\section{References}

[1] C. Christopher, P. Mardešić, and C. Rousseau, Normalizable, integrable and linearizable points in complex quadratic systems in $C^{2}$, preprint CRM (2002), to appear in J. Dynam. and Control Syst.

[2] A. A. Glutsyuk, Confluence of singular points and nonlinear Stokes phenomenon, Trans. Moscow Math. Soc., 62 (2001), 49-95.

[3] Y. Ilyashenko, Nonlinear Stokes phenomena, in Nonlinear Stokes phenomena, Y. Ilyashenko editor, Advances in Soviet Mathematics, vol. 14, Amer. Math. Soc., Providence, RI, (1993), 1-55.

[4] P. Lavaurs, Systèmes dynamiques holomorphes: explosion de points périodiques paraboliques, Ph.D. Thesis, Université de Paris-Sud, 1989.

[5] J. Martinet, Remarques sur la bifurcation nœud-col dans le domaine complexe, Astérisque 150-151 (1987), 131-149.

[6] J. Martinet, and J.-P. Ramis, Problèmes de modules pour des équations différentielles non linéaires du premier ordre, Publ. Math., Inst. Hautes Etud. Sci. 55 (1982), 63-164.

[7] J. Martinet and J.-P. Ramis, Classification analytique des équations différentielles non linéaires résonantes du premier ordre, Ann. Sci. École Norm. Sup. 16 (1983), 571-621.

[8] P. Mardešić, R. Roussarie and C. Rousseau, Modulus of analytic classification for unfoldings of generic parabolic diffeomorphisms, preprint CRM (2002).

[9] C. Rousseau, Normal forms, bifurcations and finiteness properties of vector fields, preprint CRM (2002), to appear in Proceedings of NATO Advanced Study Institute Montreal 2002, Kluwer. 